\section{Mediated responding on a multiple-choice test-only list following the acquisition of a double-function list}

\author{
KENT L. NORMAN, IRWIN P. LEVIN, and JERAL R. WILLIAMS* \\ University of lowa, lowa City, Iowa 52240
}

A mediation paradigm employing a "double-function" paired-associate list for the acquisition of the A-B and B-C chaining associations was investigated. Following acquisition of the double-function list, Ss received four multiple-choice test trials on a list containing "chaining" items for which one alternative could be chosen on the basis of a mediating chain. The tendency to choose the alternative represented by the mediating chain was significantly greater than chance in an unmixed list but was of only borderline significance for chaining items in a mixed list, for which only half the items were chaining items.

In a typical mediation chaining study, different lists (i.e., A-B and B-C paired-associate lists) are presented in succession, in order to establish associations that are presumed to mediate learning on the final test list (i.e., A-C). The present experiment explores a paradigm in which the presumed mediating associations develop during the acquisition. of a double-function paired-associate list.

A double-function list contains items that are presented as both stimuli and responses. Performance on such lists has been studied by a number of investigators (e.g., Primoff, 1938; Young, 1959, 1961). The four types of pairs of nouns making up the present list are illustrated in Table 1 and are designated as A-B, B-C, C-D, and E.F, where the words designated $A$ and $E$ each occur only as a stimulus item, D and $F$ each occur only as a response term, but $B$ and $C$ each occur as both a stimulus and a response. As a result of learning these pairs, an A-B-C chain and a B-C.D chain are assumed to develop, serving the same function as mediating chains in the three-stage chaining paradigm. The difference is that in the three-stage chaining paradigm, the pairs, $A-B, B-C$, and $A-C$, are presented on successive stages; whereas, in the present case, the $A-B$ and $B-C$ pairs are learned simultaneously. The test list in the present study corresponds to the third stage of the chaining paradigm.

Like the three-stage mediation paradigm, this paradigm is in accord with the condition of mediation theory that all mediating associations are available at the beginning of the test stage. Furthermore, there should be no differential forgetting among the various types of pairs during the test stage. The pseudomediation hypothesis proposed by Mandler \& Earhard (1964) to account for some of the mediation effects

*Now at Illinois State University, Normal, Ill. 61761. in the typical chaining paradigm argues that during B-C learning, A-B strength is reduced for $S s$ in the mediation condition, allowing $A$ in the third stage to enter into an association with $C$ more readily than in a control condition in which A-B strength is not reduced. In the present experiment, $\mathrm{A} \cdot \mathrm{B}$ and $\mathrm{B}-\mathrm{C}$ are homogeneous in material and are learned in the same list, thus reducing this possibility. An analogous procedure has been used and recommended by Earhard \& Earhard (1968). In their study completely new pairs of words were used in the test list as a within-list control for mediation effects. It is difficult, however, to justify this procedure in light of the possible effect of the interfering properties of the double-function list on the mediation items, but not on the control items. An improvement over their procedure to be incorporated into the present experiment is the use of a test stage which does not require the addition of new pairs as a control for mediation effects. Multiple-choice procedures are employed, and systematic response bias is reduced by requiring the two response alternatives for a given item to have had equal exposure on the acquisition list. For example, the stimulus DANGER is presented with the chaining response CUP and distractor BACK (see Table 1). Each of the two alternatives appears in the double-function list as both a stimulus and a response. For other chaining items on the test list, the alternatives appear only as responses in the double-function list. Because of this procedure, choice of chaining alternatives should approximate a chance level plus an increment that may be ascribed to mediated responding. Four test-only multiple-choice trials are given to see if Ss would choose the chaining alternatives more often than the distractors and if the incidence of such responding would change over trials, as would be suggested by the findings of Weaver, Hopkins, \& Schulz (1968).

A further question of interest in the present experiment is that of the effect of employing an unmixed list (where one of the alternatives could be chosen on the basis of the mediating chain for each item) vs a mixed list (where one of the alternatives could be chosen on the basis of the mediating chain for only half the items) on the test trials. Two-stage transfer studies have often found different results using different designs (e.g., A-B, A-B' and $A-B, A-B_{I}$ ). Using three-stage chaining sets, Peterson \& Cooley (1967) found that $S s$ in an unmixed condition performed better than $S s$ in a mixed condition. In fact, on test stages in the mixed condition, Ss performed more poorly on items when they were in chaining sets than when they were in control sets. Their results were discussed in terms of S's strategy and codification of the task. A mixed-list condition is included in the present study in order to see if the effect of mediated responding would be observed under these conditions. If the

Table 1

Materials for Double-Function and Test Lists

\begin{tabular}{|c|c|c|c|c|c|}
\hline \multicolumn{3}{|c|}{ Double-Function List } & \multirow{2}{*}{\multicolumn{3}{|c|}{$\begin{array}{c}\text { Test List } \\
\text { Chaining Items }\end{array}$}} \\
\hline Stimulus & \multirow{2}{*}{$\frac{\text { Response }}{\text { FOOT }}$} & $\begin{array}{c}\text { Item } \\
\text { Designation }\end{array}$ & & & \\
\hline DANGER & & $A-B$ & \multirow[b]{2}{*}{ Stimulus } & \multirow{2}{*}{$\begin{array}{l}\text { Chaining } \\
\text { Alternative } \\
\end{array}$} & \multirow{2}{*}{$\begin{array}{l}\text { Distractor } \\
\text { Alternative }\end{array}$} \\
\hline FOOT & CUP & $\mathrm{B}-\mathrm{C}$ & & & \\
\hline SON & DREAM & $E-F$ & DANGER & CUP & PAIR \\
\hline MINUTE & ISLAND & $A-B$ & MINUTE & BACK & NAME \\
\hline ISLAND & $\mathrm{BACK}$ & $\mathrm{B}-\mathrm{C}$ & FOOT & HORSE & FAMILY \\
\hline BACK & GATE & $C-D$ & ISLAND & GATE & LIFE \\
\hline INTEREST & RING & $E-F$ & \multirow{2}{*}{\multicolumn{3}{|c|}{ Nonchaining Items }} \\
\hline FACE & GROUND & $A-B$ & & & \\
\hline GROUND & NAME & B-C & Stimulus & \multicolumn{2}{|c|}{ Alternatives } \\
\hline LAKE & DRESS & $\mathrm{E}-\mathrm{F}$ & SON & GROUND & ISLAND \\
\hline SOUND & MILE & $A-B$ & INTEREST & MILE & FOOT \\
\hline MLLE & PAIR & $B-C$ & CUP & DREAM & QUESTION \\
\hline PAIR & FAMILY & $C-D$ & BACK & RING & DRESS \\
\hline VILLAGE & QUESTION & $E-1:$ & & & \\
\hline
\end{tabular}


hypothetical response of mediating using the chaining associations acquired in a previous list applies to only half of the items, it is expected that fewer chaining alternatives will be chosen than when the chaining associations are applicable to all items. Thus, in the unmixed condition, Ss should choose more chaining alternatives than would Ss in the mixed condition.

\section{METHOD \\ Lists}

The double-function list was composed of 24 Thorndike-Lorge (1944) AA nouns. The list of 16 word pairs was constructed so that two out of every six of the nouns served as both stimuli and responses (see Table 1). Within the 16 pairs there were four instances of each of four types, which may be designated A-B, B-C, C-D, and E-F. Two variations of the eight-item mixed-test list were constructed; one had as its stimulus terms the stimuli from one half of the double-function list and the other had the stimuli from the other half of the double-function list. Of the eight test items, half were nonchaining items, in which neither of the two response alternatives could be chosen on the basis of a mediating chain. The other half were chaining items in which one of the two response alternatives could be chosen on the basis of a mediating chain. For example, since DANGER was paired with FOOT, and FOOT with CUP in the double-function list, CUP was the alternative that would be chosen if the $S$ utilized the mediating chain. One half of each type of test item had stimuli that appeared only as stimuli in the double-function list, and half had stimuli that appeared as both stimuli and responses. In all cases the two alternatives for a given item had been of equal exposure. In each of the two variations of the four-item unmixed test list, the items were identical to the chaining items used in the mixed list.

\section{Procedure}

The double-function list was presented as a paired-associate task-using a study-test method. Three different orders of the study list were typed on a tape for presentation on a Stowe memory drum at a 2:3-sec rate for study and test intervals, respectively, with a 3 -sec intertrial interval. Guessing was encouraged to eliminate omissions. The list was performed to a criterion of one errorless trial. After a short interval, four orders of the mediation test list were presented on a tape, and a S-paced rate for presenting items was adopted. The instructions for this stage required the $S$ to say the stimulus word and then pick one of the two response words that he thought might be "correct," remembering the first list that he had learned. He was required to give the number ( 1 or 2 , representing the left-right position) of that word. He was further told that he should change his answer in later presentations of the list only if he had a reason.

\section{Subjects}

The $48 \mathrm{Ss}$ completing this experiment had been assigned randomly to two groups of equal size for mixed and unmixed presentation of the test list, with the restriction that half were male and half female in each group. The Ss participated to fulfill a course requirement in an introductory psychology class. Because of time limitations, 14 Ss were discarded due to the inability to reach acquisition criterion within 27 trials.

\section{RESULTS \\ Acquisition of the}

Double-Function List

The mean number of trials to criterion on the 16-item list was .15.9, with a standard deviation of 6.25 for the mixed group, and was 16.7 for the unmixed group, with a standard deviation of 5.50 . The two means did not differ significantly, $F(1,46)<1.00$.

\section{Test List}

The mean proportion of chaining alternatives chosen by $\mathrm{Ss}$ in the unmixed list condition was .634. This was significantly greater than chance (.50), $\mathrm{t}(23)=3.26, \mathrm{p}<.01$. The mean proportion of chaining alternatives chosen in the mixed condition was .580 , which differed from chance to a degree of only borderline significance using a one-tailed test, $t(23)=1.67, .05<\mathrm{p}<.10$. A test for the predicted difference between the means for the mixed and unmixed conditions, however, was not significant, $t(46)<1.00$.

An analysis of variance, which included trials as a factor, revealed no significant change in the proportion of chaining alternatives chosen over trials. The means on Trial 4 were virtually the same as the means of Trial 1. Analyses of individual items revealed no significant change over trials for any item. No significant difference in mean proportion of choices of chaining alternatives was found between items in which the A-B-C chain and items in which the B-C-D chain were employed. $A$ sequential analysis of conditional probabilities indicated a greater-than-chance tendency for the $S$ to repeat the response given on the immediately preceding trial. This was statistically significant for all types of items ( $p<.001$ in each case) and is in accord with the instructions.

\section{DISCUSSION}

The present experiment shows a mediated facilitation effect following the learning of a double-function list. This finding cannot be explained in terms of an interference theory like that of Earhard \& Earhard (1968), since both A-B and B-C associations were learned to criterion in the same list and would therefore suggest mediated responding. It may be assumed that when the stimulus in the test list is presented to the $S$, he samples some mediating chain leading to either Alternative 1 or 2 . If the mediating chain sampled is a learned association from the double-function list, it is assumed that he will choose the chaining alternative with a probability of 1.0 . If the mediating chain sampled is an unknown preexperimental or idiosyncratic association, then the expected probability of choosing the chaining alternative would be .50 . Performance on the test list is in agreement with the notion that the associations built up during acquisition of the double-function list serve to increase the probability of sampling the experimentally induced mediation chain leading to the choice of the chaining alternative.

Weaver, Hopkins, \& Schulz (1968) found, in a three-stage chaining paradigm, a significant increase in the incidence of mediated responding of $\mathrm{Ss}$ in the chaining condition over test-only trials on the A-C stage. Though the test trials used were similar, no such systematic change over trials was observed. The present list, however, differs in one major respect-it was S-paced. Such results might indicate that the sampling of mediating chains takes place in time and that given enough time the $\mathrm{S}$ may be able to sample on the very first test trial all the experimentally induced mediating chains that he will subsequently use.

The effect of mixed vs unmixed presentation was inconclusive. A significant difference was not found between the incidence of mediated responding in the mixed and unmixed conditions. However, reliable mediation effects were observed only in the unmixed condition. A further study of the difference in responding between the mixed and unmixed conditions is planned.

\section{REFERENCES}

EARHARD, B., \& EARHARD, M. Interference, strategies, and the mechanism of mediation. Journal of Experimental Psychology, 1968 , $78,216-227$.

MANDLER, G., \& EARHARD, B. Pseudomediation: Is chaining an artifact? Psychonomic Science, 1964, 1, 247-248.

PETERSON, M. J., \& COOLEY, R. Effects of unmixed and mixed presentation upon verbal mediating responses. Journal of Verbal 
Learning \& Verbai Beńavior, $1967,6,328-334$

PRIMOFF, E. Backward and forvard association as an organizing act in serial and in paired associate learning. Joumal of Psychology, $1938,5,375-395$.

THORNDIKE, E. L., \& LORGE, I. The teacher's word book of 30,000 words. New York: Columbia University, 1944.

WEAVER, G. E., HOPKINS, R, H., \& SCHULZ, R. W. The $A-B, B-C, A-C$ mediation paradigm:
A-C performance in the absence of study trials. Journal of Experimental Psychology, 1968 , $77,670-675$

YOLNG, R. K. A comparison of two me thods of learning serial associations. American Joumal of Psychology, 1959, 72, 554-559.

YOUNG, R. K. Paired-associate learning when the same items occur as stimuli and responses. Journal of Experimental Psychology, 1961, $61,315-318$.

\section{Effects of proportion of positive instances and degree of restriction on the induction of a principle*}

\author{
DENNIS DERVIN and KENNETH DEFFENBACHER† \\ University of Nebraska at Omaha, Omaha, Nebr. 68101
}

Ss attempted to discover a principle by which numbers and letters were paired. Seven groups of Ss varied on two dimensions, proportion of positive instances sampled and degree of restriction in selecting instances for test. Ss who sampled a higher proportion of positive instances were superior to those sampling a lower proportion. Ss who were unrestricted in their selection of instances, because they sampled a higher proportion of positive instances, performed better than Ss who were restricted and sampled a lower proportion. Finally, when both restricted and unrestricted groups were equated for proportion of positive instances sampled, performance was similar.

A number of studies have investigated the effects of the degree of restriction placed upon $\mathrm{S}$ in his attempt to discover the solution to a problem (Huttenlocher, 1962; Duncan, 1964; Hunt, 1965; Murray \& Gregg, 1969). Both the Huttenlocher and Murray and Gregg studies utilized single-cue concept problems, which $S$ solved either under a selection (unrestricted in instance selection) or a reception (restricted in instance selection) paradigm. Each study found that the reception group solved the problems more rapidly than did the selection group. Hunt's Ss solved considerably more difficult problems than those of the previous two studies; Ss had to solve either conjunctive concept problems or to learn to recognize legitimate letter strings generated by a finite-state grammar. Hunt found that Ss solving under selection conditions were superior to those solving under reception conditions. The results of

*This research was carried out by the senior author at the University of Nebraska at Omaha in partial fulfillment of the requirements for the MA degree.

† Requests for reprints should be sent to Kenneth Deffenbacher, Department of Psychology, Lniversity of Nebraska at Omaha, Omaha, Nebraska 68101 the three studies discussed thus far would seem to suggest that freedom in instance selection is advantageous only when the task is a difficult one. Duncan's study does not appear to fit this pattern. He found the same result as did Hunt, yet his task would appear to be less difficult; Ss had to discover E's principle for pairing numbers and letters.

There may be a confounding factor in Duncan's study, however. Ss who were unrestricted in instance selection may have been sampling a higher proportion of positive instances than were $S s$ in the partially and totally restricted groups. Duncan arbitrarily imposed a 50:50 split of positive and negative instances on $\mathrm{Ss}$ in the partially and totally restricted groups. As Ss in the unrestricted group were free to choose any instance they wished, their proportion of positive instances could thus deviate significantly from the 0.50 proportion imposed on the other groups. Hovland \& Weiss (1953) and Haygood \& Stevenson (1967) have presented evidence showing that naive Ss utilize positive instances much more efficiently than they do negative instances.

The present study seeks to replicate Duncan's study and then to show that his unrestricted group's greater problem-solving efficacy was due, not to the restriction variable, but rather to the higher proportion of positive instances that they sampled.

Seventy Ss were drawn from an introductory psychology course at the University of Nebraska at Omaha as a part of a course requirement. Ten Ss were unsystematically assigned to each of seven treatment groups.

In order to test the contention that Duncan's results were at least partially an artifact of the proportion of positive instances sampled (the unrestricted group sampling a higher proportion than the restricted groups), it was necessary to run the unrestricted group (U group) first. This was necessary in order to determine the mean proportion of positive instances sampled by this group.

Of the seven groups, four were replicates of Duncan's conditions. These four groups included the $U$ group, two partially restricted groups, and one totally restricted group. In Group RN (restricted numbers), $S$ was allowed to choose any letter he wished but was told which number to write down. Similarly, Group RL (restricted letters) chose any number but was told which letter to record. Group RNL (restricted numbers and letters) was told which number and which letter to record. All three groups (RN, RL, and RNL) had the same 50:50 split of positive and negative instances imposed on them, as was the case in Duncan's study. These groups are referred to, respectively, as RN50, RL50, and RNL50.

The remaining three groups operated under the same conditions as those stated above. However, these groups sampled the same proportion of positive instances as that sampled by the $\mathrm{U}$ group. They are referred to as RN (U\%), RL (U\%), and RNL (U\%). These groups were added to test the contention that Duncan's results were due, in part at least, to the proportion of positive instances sampled.

The task was the same as in Duncan's study. By creating number-letter pairs. $S$ attempted to discover the principle: "Any of the even numbers $(2,4,6,8)$ may be paired with any of the letters A.E; any of the odd numbers $(1,3,5,7)$ may be paired with any of the letters F-J." Each trial consisted of the creation of a number-letter pair, followed by feedback from $E$ as to whether the instance was or was not an example of the principle. $\mathrm{S}$ was allowed to work at his own pace and was allowed to guess at the principle at any time. After each guess, $E$ informed $S$ whether the hypothesis was correct, partially correct, or wrong. If $S$ had not stated the principle correctly after $25 \mathrm{~min}$, he was stopped and 